\title{
WLAN Performance Improvisation by Fine Tuning IEEE 802.11 Parameters
}

\author{
Rajan Vohra \\ Dept. of Electronics \\ Technology \\ Guru Nanak Dev \\ University \\ Amritsar, Punjab, India
}

\author{
R.S.Sawhney \\ Dept. of Electronics \\ Technology \\ Guru Nanak Dev \\ University \\ Amritsar, Punjab, India
}

\author{
Shipra Nayyar \\ Dept. of Electronics \\ Technology \\ Guru Nanak Dev \\ University \\ Amritsar, Punjab, India
}

\author{
Sunandika Mann \\ Dept. of Information \\ Technology \\ KSOU, Mysore, India
}

\begin{abstract}
Wireless networks has biggest constraint of limited bandwidth in comparison to wired networks but in spite of this constraint wireless networks are becoming popular day by day on account of their flexibility, mobility as well as inexpensive physical medium (air). Wireless local area networks (WLANs) are error-prone as well as fragile. The commuters feel annoyed when all of sudden communication cuts off and transmission of packets fails. Hence, it becomes an obligation to improve WLAN performance metrics. We investigated throughput and delay performance of WLANs using network simulation tool OPNET concentrating on the IEEE 802.11 Mac layer parameters, and suggest that Wireless LAN performance can be improved by fine tuning parameters such as fragmentation threshold, request to send (RTS) thresholds and buffer size. The main objective of this paper is buffer size manipulation to reduce load and delays, leading to performance improvement.
\end{abstract}

\section{General Terms}

Buffer size, delay, load

\section{Keywords}

WLAN, OPNET, Media Access Delay, Throughput.

\section{INTRODUCTION}

A mobile ad hoc network is a self-organizing network comprising wireless mobile nodes that move around arbitrarily and can able to communicate among themselves using wireless radios, without the aid of any pre-existing infrastructure [9]. Each participating mobile node can act as sender, receiver and even as a router at the same time and able to build, operate and maintain these networks [10]. Wireless technology has helped to simplify networking by enabling multiple computer users to simultaneously share resources in a home or business without additional or intrusive wiring. These resources might include a broadband Internet connection, network printers, data files, and even streaming audio and video.

Compared to traditional LAN technologies, WLAN technology has the advantage of mobility for Internet access. WLAN users can obtain transmission rates up to $11 \mathrm{Mbps}$ and $54 \mathrm{Mbps}$ and will be up to or over $100 \mathrm{Mbps}$ [1], which is of special interest for low cost and a reasonable rate. The introduction of these technologies created a need to incorporate it to network classes. Computer simulation is becoming increasingly popular among computer network researchers for performance modeling and evaluation of computer and telecommunication networks. This popularity is due to the availability of various sophisticated and powerful simulators, and also because of the flexibility in model construction and validation offered by simulation. While various network simulators (both open source and commercial) exist for modeling and performance evaluation of communication networks. With the existence of a wide variety of network simulation tools, OPNET $^{\mathrm{TM}}$ simulation tool is the first choice of industry since it is easy to train on and a powerful tool to simulate up to date network technologies. [2]

\section{RELATED WORK}

There exist a wide different techniques or methods for evaluation and enhancements of wireless Local area network that has been postulated from both analytic and simulation-based studies on mobile communication networks. This section describes a sampling of these methods that have been designed

specifically for enhancements and performance evaluation of Wireless Local Area Networks. Some previous research mainly focused on improving the performance via network layer and performance optimization has been shown via a series of simulation tests with different parameters such as RTS/CTS threshold. To reduce throughput reduction owing to hidden stations, 802.11 specifies as an option the exchange of Request-toSend/Clear-to-Send (RTS/CTS) frames. Before transmitting a data frame, a station may transmit a short RTS frame, which must be followed by a CTS frame transmitted by the receiving station. The RTS/CTS mechanism is very effective in terms of system performance, especially when large packets are considered, as it reduces the length of the frames involved in the contention process, can be found in [11], in details. Several modifications of the IEEE 802.11 DCF access method have been proposed recently to improve the performance of wireless LANs. Up to now, such proposals have only been compared under ideal channel conditions [12]. In $[13,14,15]$ several investigations have been made with different methods for improving the performance of WLANs, Such as Adaptive (rather than basic) back-off algorithms in the MAC Layer, proxy approaches in the link-layer, such as snoop protocol, and TULIP , Split-connection approaches, such as I-TCP or MTCP and Other link-layer approaches, such as AIRMAIL. In these papers a survey research literature dealing with improving performance on various wireless network layers and the OPNET implementations have been described via several simulation scenarios.

This paper is focused on the studies of wireless Local Area networks in a simulated environment using OPNET $^{\mathrm{TM}}$ IT Guru Academic IT Guru Academic Edition (2007). [2] In this paper, the work has been further extended and reported performance enhancement of WLAN by customizing IEEE 802.11 layer related parameters. We demonstrate that WLAN performance can be improved by tuning parameters such as fragmentation threshold, request to send (RTS) thresholds and buffer size. Customizing buffer size parameter to use the values specified in the standards will reduce delays, and reduce load on nodes.[3][4][5]

\section{OUR APPROACH}

OPNET is a tool used to simulate the way networks run. In this paper, we have carried out a comparative study on delay, throughput and load in wireless LAN configured for video conferencing. We have chosen simulative tool- OPNET for our research because of the several benefits it offers over the other contemporary tools available. OPNET IT Guru is a powerful 
communication system simulator developed by OPNET Technologies [8]. OPNET IT Guru 9.1 assists with the testing and design of communications protocols and networks by simulating network performance for wired and/or wireless environments. The OPNET tool provides a hierarchical graphical user interface for the definition of network models. A network is constructed by graphically connecting network nodes via communications links. OPNET IT Guru comes with an extensive model library, including application traffic models (e.g., HTTP, FTP, E-mail, Database), protocol models (e.g., TCP/IP, IEEE 802.11 b, Ethernet), and a broad set of distributions for random variant generation. Another advantage of using OPNET is that it is being extensively used and there is wide confidence in the validity of the results it produces. OPNET enables realistic analysis of performance measures and the effectiveness of wireless network design techniques.

\section{SCENARIOS AND SETTINGS}

In this section, we consider the case of two scenarios in which two independent wireless LANs workstations are connected. Here, these workstations are configured for the video application only as in figure 1. In scenario 1 and 2 workstations are operated at $11 \mathrm{Mbps}$ data rate. Two different scenarios and settings have been considered to optimize the network.

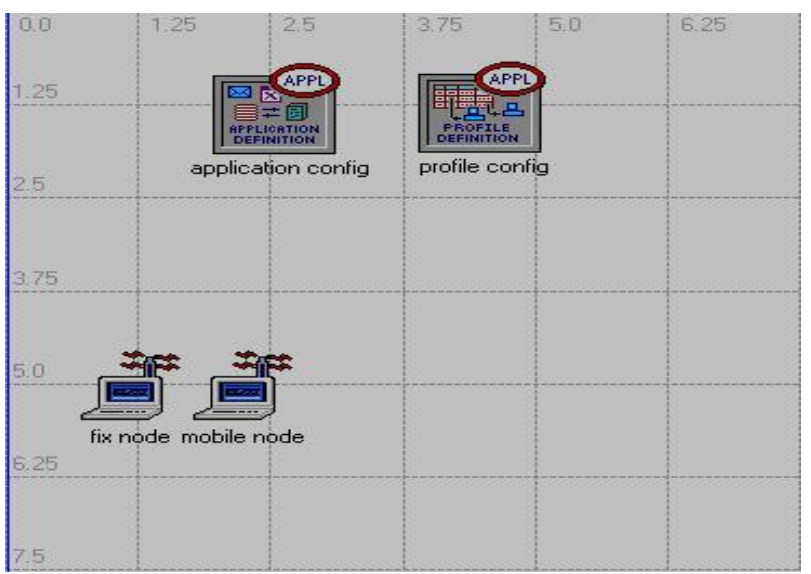

Figure 1. WLAN scenario configured for video application

Table 1 shows the parameters, which are used in different scenarios for simulation. Parameters show that we use PHY characteristics as Direct sequence, buffer size (bits) 1024kbits and 64 Kbits for scenario 1 and 2. Bandwidth used for the WLAN for scenario 1 and scenario 2 is $11 \mathrm{Mbps}$ and $11 \mathrm{Mbps}$.

Table 2 shows the wireless Lan traffic generation parameters for both the scenario. Operation Mode used in the scenario is serial (ordered), start time (seconds) are uniform $(100,110)$, repetition pattern in the scenarios is serial, numbers of repetition is constant and rest of the parameters used are listed in table below:
Table 1. Simulation scenario parameters

\begin{tabular}{|l|c|c|}
\hline \multicolumn{1}{|c|}{ Parameter(s) } & Scenario 1 & Scenario 2 \\
\hline Address & Auto Assigned & Auto Assigned \\
\hline $\begin{array}{l}\text { WLAN } \\
\text { bandwidth (bps) }\end{array}$ & $11 \mathrm{Mbps}$ & $11 \mathrm{Mbps}$ \\
\hline $\begin{array}{l}\text { PHY } \\
\text { Characteristics }\end{array}$ & \multicolumn{2}{|c|}{ Direct Sequence } \\
\hline Slot time (s) & \multicolumn{2}{|c|}{5.0 E-05 } \\
\hline $\begin{array}{l}\text { Packet Reception- } \\
\text { Power Threshold } \\
\text { (W) }\end{array}$ & \multicolumn{2}{|c|}{7.33 E-14 } \\
\hline $\begin{array}{l}\text { Short Retry Limit } \\
\text { (Attempts) }\end{array}$ & \multicolumn{2}{|c|}{64000} \\
\hline $\begin{array}{l}\text { Long Retry Limit } \\
\text { (Attempts) }\end{array}$ & 1024000 & Video conference \\
\hline Buffer size(bits) & Video conference \\
\hline $\begin{array}{l}\text { Application } \\
\text { supported profile }\end{array}$ & \multicolumn{2}{|c}{} \\
\hline $\begin{array}{l}\text { Max Receive } \\
\text { Lifetime (secs) }\end{array}$ &
\end{tabular}

Table 2. Wireless LAN Traffic Generation Parameters

\begin{tabular}{|l|l|}
\hline \multicolumn{1}{|c|}{ Attribute } & \multicolumn{1}{c|}{ Value } \\
\hline Duration & End of simulation \\
\hline Repeatability & Once at Start Time \\
\hline Operation Mode & Serial (ordered) \\
\hline Start Time (seconds) & constant (300) \\
\hline $\begin{array}{l}\text { Inter-repetition (100,110) } \\
\text { (seconds) }\end{array}$ & constant (30) \\
\hline $\begin{array}{l}\text { Number of Repetitions } \\
\text { Repetition Pattern }\end{array}$ & Serial \\
\hline
\end{tabular}

\section{SIMULATION EVALUATION}

Wireless Local Area Network scenario was created and configured and finally simulations were performed using a versatile network simulation tool OPNET. OPNET 802.11b PHY module was used as a standard with maximum data rate up to $11 \mathrm{Mbps}$ using direct sequence spread spectrum for videoconferencing applications. The packet size is default. When workstations are operated at $11 \mathrm{Mbps}$ in scenario 1 and 2 , figure 2 shows that throughput shows transiency for first seven minutes in both cases and reaches steadystate after that. The simulated values at seven minute are $2000 \mathrm{Kbps}$ in case of buffer size $64 \mathrm{Kbits}$ and $4300 \mathrm{Kbps}$ in case of 1024 Kbits buffer size. Hence it is evident that there is increase in throughput when buffer size has been increased. 


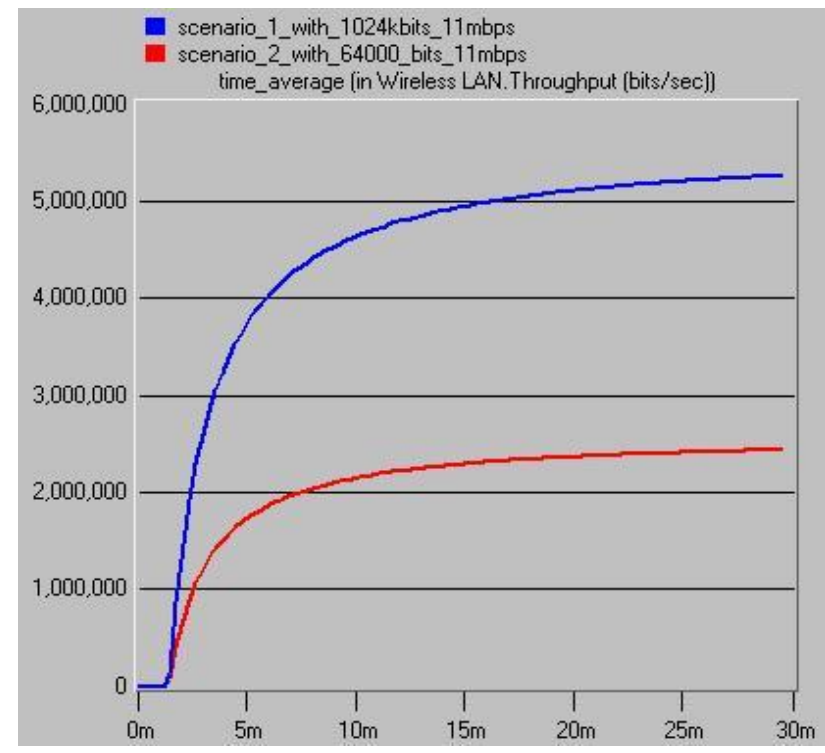

Figure 2 WLAN throughput (bits/sec) for 11 Mbps scenarios

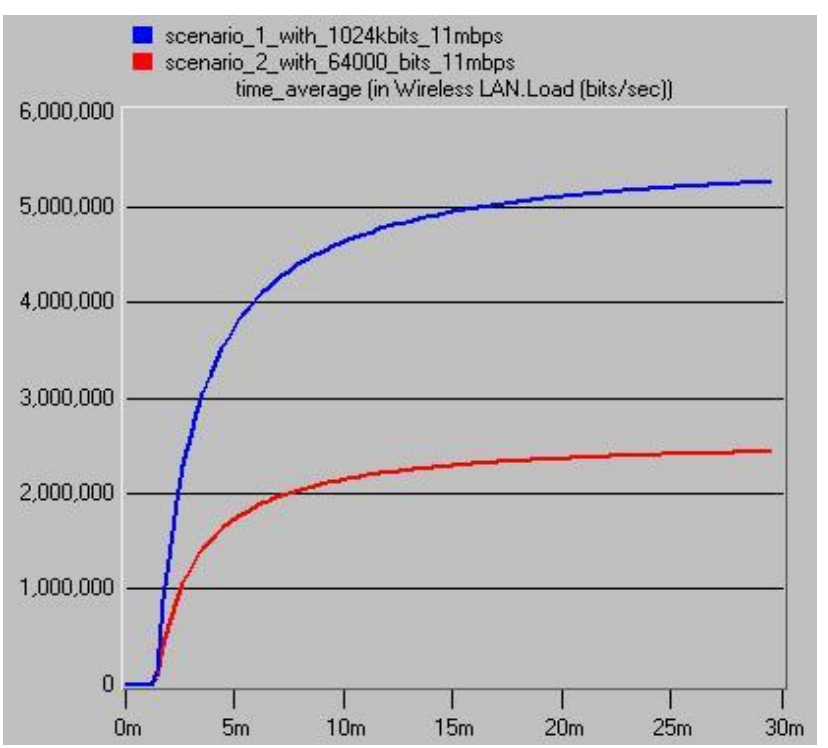

Figure 3 WLAN load(bits/sec) for 11 Mbps scenarios

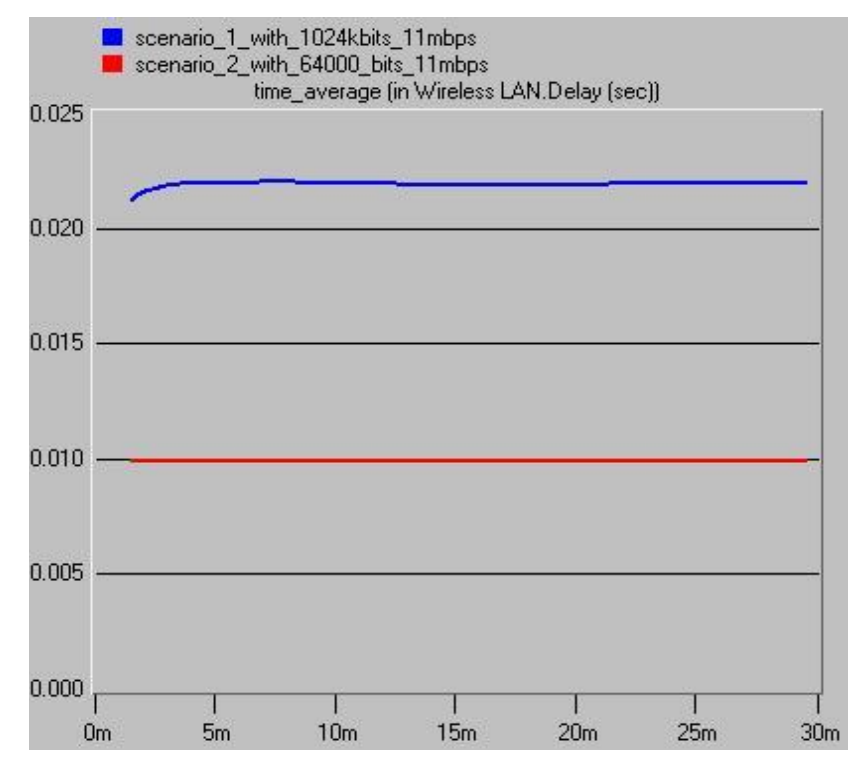

\section{Figure 4 WLAN delay(sec) for 11 Mbps scenarios}

In figure 3 WLAN load (bits/sec) has been plotted for both the scenarios and we have observed that WLAN load (bits/sec) on the workstations is $2300 \mathrm{Kbps}$ for $64 \mathrm{kB}$ buffer size, while this value is $5000 \mathrm{kbps}$ for the $1024 \mathrm{kB}$ buffer size, which indicate that the WLAN load decreases when the buffer size is reduced.

We observed the results with the customized paramters of WLAN and compared these with the previous scenarios, where customization was not done. Figure 4 and 5 shows WLAN delay (sec) and Media Access Delay (sec) results which show a marginal decrease in both the delays with time. We considered results at 10 minutes mark and observed that the value of delay and Media access delay in 64kbits scenario is $0.010 \mathrm{sec}$ and $0.008 \mathrm{sec}$ respectively but in case of 1024 kbits scenario it is 0.022 and 0.020 sec. The observed difference of $0.012 \mathrm{sec}$ shows the improvement in case of 64000 bits scenario.

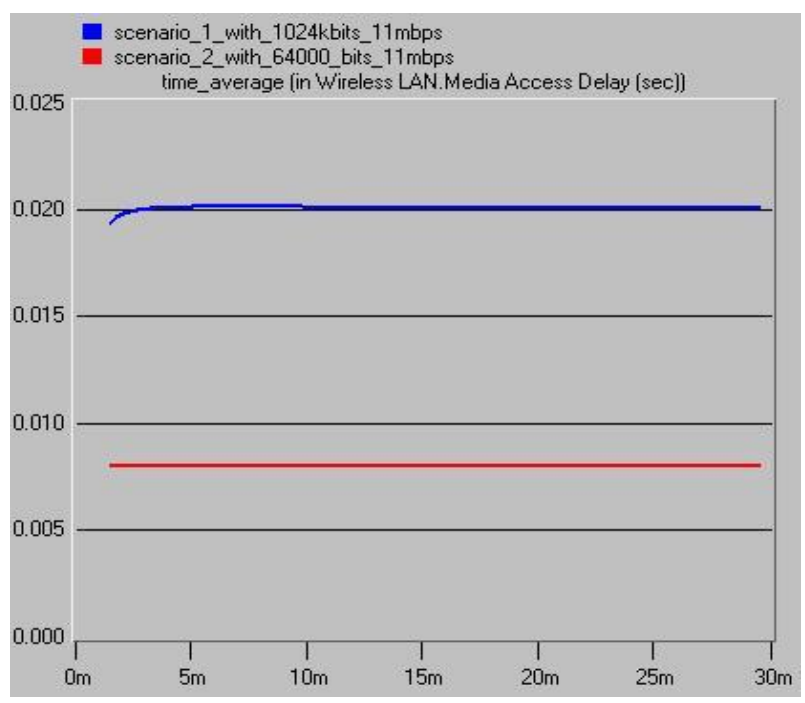

Figure 5 WLAN Media Access Delay (sec) for scenarios

\section{CONCLUSIONS}

This paper investigates the delay, throughput, media access delay and load for 64kbits and 1024 Kbits scenarios and their comparison thereof. Low throughput in case of $64 \mathrm{kbits}$ scenario of around $2000 \mathrm{Kbps}$ have been reported, while it is high in case of 
1024 Kbits scenario of around $4300 \mathrm{kbps}$. A high delay in case of $1024 \mathrm{kbps}$ scenario of the order of $0.022 \mathrm{sec}$ has been reported, while it is lowest in case of 64kbps measuring $0.010 \mathrm{sec}$ respectively. Further, the results demonstrate measurements of load on workstations for both the scenarios. In case of Media access delay the difference of around $0.012 \mathrm{sec}$ has been reported and it is less in case of 64kbits scenario when compared to 1024 kbits scenario. To conclude, it is reported that the 64 Kbits scenario offer less load, WLAN delay, media access delay and throughput in comparison to $1024 \mathrm{Kbits}$ scenario. Overall performance of the WLAN network with 64 Kbits buffer size can be increased by compensating small throughput.

\section{REFERENCES}

[1] G. Holland and N. Vaidya, (2002), "Analysis of TCP Performance over Mobile Ad Hoc Networks," 5th Annual Int'l. Conf. Mobile Comp. And Net, pp. 275-288, 2002 [Academic Publishers. Manufactured in The Netherlands].

[2] E. Baburaj and V. Vasudevan (2008), "An Intelligent Multicast Ad-hoc On demand Distance Vector Protocol for MANETs" Journal of Networks, Vol. 3, No. 6, June 2008.

[3] Soliman A. Al-Wabie (2002), "The New Wireless Local Area Networks (WLAN's) Standard”, University of Maryland.

[4] IT Guru Academic Edition (2007),OPNET Technologies www.opnet.com/university_program/itguru_academic_edition

[5] Ms. Amardeep Kaur1, Dr. Sandip Vijay2, Dr. S.C.Gupta3 "Performance Analysis and Enhancement of IEEE 802.11 Wireless Local Area Networks" Vol. 9 Issue 5 (Ver 2.0), pp.130. January 2010.

[6] Q. Ni, I. Aad, C. Barakat, and T. Turletti, "Modeling and Analysis of Slow CW Decrease for IEEE 802.11 WLAN". 14th IEEE Proceedings on Personal, Indoor and Mobile Radio Communications, IEEE PIMRC 2003, vol. 2, pp. 1717-1721. Beijing, September 2003.

[7] Dr. Mayyada Hammoshi, Mrs. Razan Alani, "A Proposed Model to Implement Load and Throughput of WLAN
Implemented as Wi-Fi System", JCIT: Journal of Convergence Information Technology, Vol. 5, No. 7, pp. 100 $\sim 111,2010$

[8] M. Heusse, F. Rousseau, R. Guillier, and A. Duda, "Idle sense: An optimal access method for high throughput and fairness in rate diverse wireless LANs," in Proc. of ACM SIGCOMM 2005, vol 35; NUMB 4, pages 121-132, August 2005.

[9] Mrs. Razan Al-Ani, "Simulation and Performance Analysis Evaluation for Variant MANET Routing Protocols", IJACT: International Journal of Advancements in Computing Technology, Vol. 3, No. 1, pp. $1 \sim 12,2011$.

[10] Wlan_lab_script_1_2 from http:// www.comnets.unibremen.de/ mms/wlan_lab_script_1_2.pdf

[11] Walid Hneiti(2006),"Performance Enhancement of Wireless Local Area Networks" Amman Arab University for Graduate Studies, Jordan.

[12] Manju Sharma and Manoj, "Comparative Investigation on Throughput and Client Response Time for a Switched and Routed Wireless LAN based on OPNET" Presented and published in the proceedings of National Conference on "Emerging Trends in "Computing and Communication (ETCC-07) at national institute of Technology, Hamirpur, (HP), India during July 27-28, 2007, pp 436-440.

[13] OPNET Technologies, www.opnet.com

[14] IEEE, "IEEE standards for information technology telecommunications and information exchange between systems - local and metropolitan area network - specific requirements - part 11: Wireless LAN medium access control (MAC) and physical layer (PHY) specifications," 1999.

[15] Mohammad Hussian Ali and Manal Kadhim Odah, Simulation Study Of 802.11b DCF Using OPNET Simulator", Eng. \& Tech. Journal, Vol. 27,No6 , pp. 1108-1117. 2009. 